\title{
Treatment of Rodent Liver Tumor With Combretastatin A4 Phosphate
}

\author{
Noninvasive Therapeutic Evaluation Using Multiparametric Magnetic \\ Resonance Imaging in Correlation With Microangiography and Histology
}

\author{
Huaijun Wang, MD, * Xihe Sun, MD, *广 Feng Chen, MD, * Frederik De Keyzer, MSc, * Jie Yu, MD, * \\ Willy Landuyt, PhD, $\S$ Vincent Vandecaveye, MD, * Ronald Peeters, PhD, * Hilde Bosmans, PhD, * \\ Robert Hermans, MD, PhD, * Guy Marchal, MD, PhD, * and Yicheng Ni, MD, PhD*
}

\begin{abstract}
Objectives: To document tumoricidal events after intravenous administration of a vascular targeting agent combretastatin A-4-phosphate (CA4P) in rodent liver tumors by using multiparametric magnetic resonance imaging (MRI) in correlation with microangiography and histopathology.

Materials and Methods: Thirty rhabdomyosarcomas of 8 to $14 \mathrm{~mm}$ in diameter were obtained 16 days after implantation in liver lobes of 15 rats. Using a $1.5 \mathrm{~T}$ magnet and a 4-channel wrist coil, T2-weighted imaging (T2WI), pre- and postcontrast T1-weighted imaging (T1WI), diffusionweighted imaging (DWI), and dynamic susceptibility imaging (DSI) with relative blood volume (rBV) and flow (rBF) maps were acquired at baseline, 1 hour, 6 hours, and 48 hours after iv injection of CA4P at $10 \mathrm{mg} / \mathrm{kg}$ and vehicle in 9 treated and 6 control rats, respectively. In vivo data including signal intensity (SI), tumor volume, apparent diffusion coefficient (ADC), $\mathrm{rBV}$, and rBF were correlated with ex vivo microangiographic and histopathologic findings.
\end{abstract}

Results: CA4P-treated tumors $(\mathrm{n}=18)$ grew slower than those (n $=12)$ of controls $(P<0.05)$, with vascular shutdown evident on CE-T1WI at 1 hour but more prominent at 6 hours. However, enhanced rim occurred in the periphery 48 hours after treatment, indicating neovascularization. ADC map enabled distinction between necrotic and viable tumors. DSI-derived tumoral $\mathrm{rBV}$ and $\mathrm{rBF}$ decreased significantly at 1 hour through 6 hours and partly recovered at 48 hours. SI-time curve reflected diverse therapeutic responses between tumor and liver. MRI findings were verified by ex vivo techniques.

Conclusions: Clinical MRI allowed monitoring of CA4P-related vascular shutdown, necrosis, and neovascularization of liver tumors in rats. Single dose of CA4P seemed insufficient for tumor eradication because of evident peripheral residue and recurrence.

Received May 18, 2008, and accepted for publication, after revision, September $11,2008$.

From the *Section of Radiology, Department of Medical Diagnostic Science, University of Leuven, Leuven, Belgium; †Department of Radiology, Affiliated Hospital of Weifang Medical University, Shandong, China; $¥$ Department of Radiology, Zhongda Hospital, Southeast University, Nanjing, China; and §Laboratory of Experimental Radiobiology and Oncology, Faculty of Medicine, University of Leuven, Leuven, Belgium.

This work was partially supported by the grants awarded by Fonds voor Wetenschappelijk Onderzoek-Vlaanderen (FWO Vlaanderen) Impulsfinanciering project (ZWAP/05/018), Geconcerteerde Onderzoeksactie (GOA) of the Flemish Government, OT project (OT/06/70) MoSAIC, the K.U. Leuven Molecular Small Animal Imaging Center (KUL EF/05/08), and a EU project Asia-Link CfP 2006-EuropeAid/123738/C/ACT/Multi-Proposal 128-498/111.

Reprints: Yicheng Ni, MD, PhD, Section of Radiology, Department of Medical Diagnostic Science, University Hospitals, University of Leuven, Herestraat 49, bus 7003, B-3000 Leuven, Belgium. E-mail: Yicheng.Ni@med. kuleuven.be.

Copyright (C) 2008 by Lippincott Williams \& Wilkins

ISSN: 0020-9996/09/4401-0044
Key Words: combretastatin A-4-phosphate (CA4P), magnetic resonance imaging (MRI), animal, liver, tumor

(Invest Radiol 2009;44: 44-53)

In cancer drug developments, 2 antivascular approaches have shown promises in experimental and clinical oncology: antiangiogenic agents inhibit the proliferation of tumoral neovasculature, whereas vascular targeting agents (VTAs) selectively destroy existing tumor vessels, both exerting anticancer effects through deprivation of tumor blood supply and starvation of malignant cells. ${ }^{1}$ These drugs have undergone clinical trials in different phases. ${ }^{2}$

Among the VTAs, combretastatin A4 (CA4) is extracted from the bark of the South African bush willow tree, Combretum caffrum. It has a high affinity for tubulin, the only cytoskeleton to maintain tumoral endothelium in flat shape. Upon intracellular uptake and molecular interactions, CA4 makes tubulin depolymerized, which in turn leads to destabilization or deformation of the lining endothelial cells, subsequent occlusion of intratumoral microcirculation, and consequent tumor destruction. ${ }^{1,3,4}$ As a more water-soluble prodrug of CA4, CA-4-phosphate (CA4P) is metabolized into the active CA4 after intracellular uptake. ${ }^{4-6}$ It has been demonstrated that CA4P induces blood flow shutdown in tumors within minutes after administration, resulting in pronounced tumor necrosis, ${ }^{7-9}$ whereas normal tissues are much less affected. ${ }^{7,9,10}$ This selective effect can be attributed to the assumption that actin as another cytoskeleton is available in normal endothelium but deficient in tumoral endothelium. Therefore, the normal vasculature is much less susceptible to CA4 than the abnormal one. ${ }^{1-10}$ In addition to the treatment of various tumors in extensive preclinical and clinical studies, ${ }^{9,11-15}$ CA4P has shown potentials in prevention and modulation of nonmalignant disorders such as ocular neovascularization. ${ }^{16}$

As a widely applied noninvasive imaging modality, magnetic resonance imaging (MRI) seems ideal for in vivo assessment of the antitumor effect of VTAs through documentation of morphologic and functional alterations by using multiparametric sequences including diffusion weighted imaging (DWI) and dynamic contrast-enhanced MRI (DCE-MRI). ${ }^{17-22}$

In the previous studies on CA4P, almost all animal models were produced by subcutaneous inoculation of tumor cell suspension or implantation of tumor tissue fragment in the flank region, mainly for easier observation and measurement of tumor growth as a therapeutic response, and almost all these studies applied intraperitoneal administration of CA4P, ${ }^{7-9,23,24}$ which differ obviously from the clinical scenarios. ${ }^{13,15}$ Besides, MRI examinations in most of these studies were mainly for acquiring morphologic information and performed with small-bore dedicated animal scanners of high magnetic field strength $(>4.7 \mathrm{~T})$. 
Recently, a rat model of liver rhabdomyosarcoma (R1) has been introduced and characterized using a clinical $1.5 \mathrm{~T}$ system, which allows multiparametric assessment of malignant versus normal tissues with updated software, hence more translational to the applications in human patients. ${ }^{25}$ The aim of the current study is to validate the usefulness of this newly developed platform for preclinical evaluation of anticancer drugs with the following 3 hypotheses: (1) liver tumors in rats could be treated by intravenously administered CA4P; (2) the therapeutic effects could be noninvasively monitored and comprehensively evaluated at a clinical MRI unit, and (3) the in vivo imaging data could be supported by postmortem gold-standard techniques including microangiography and histopathology.

\section{MATERIALS AND METHODS}

\section{Tumor Model}

This study was approved by the local ethical committee for animal care and use. The animals were maintained and provided by the laboratory of experimental oncology in our institute. An adult rat with R1 growing in the flank was used as a donor. The syngeneic transplantation of R1 tumor provides a highly reproducible experimental tumor model with biologic features and therapeutic responses similar to those of human malignancies. ${ }^{25,26}$

Fifteen male WAG/Rij rats about 14 weeks old, weighing 280 to $300 \mathrm{~g}$, received implantation of R1 in the liver. Briefly, rats were anesthetized with an intraperitoneal injection of pentobarbital (Nembutal; Sanofi Sante Animale, Brussels, Belgium) at $60 \mathrm{mg} / \mathrm{kg}$. After midline laparotomy, 2 freshly harvested $\mathrm{R} 1$ pieces of 1 to $2 \mathrm{~mm}^{3}$ were inserted into the apertures made in left and right lobes to induce bifocal liver tumors. ${ }^{25}$ The tumor growths were followed with MRI every 2 days after 1 week after the implantation.

\section{Experimental Design}

The experiment recruited a total of 15 rats with $30 \mathrm{R} 1$ liver tumors (2 from each rat) of 8 to $14 \mathrm{~mm}$ in diameter. All rats underwent a baseline MRI examination before the treatment. CA4P (Oxigene Inc., Watertown, MA) diluted in 0.3-mL phosphate buffered saline (PBS) was injected via penile vein at a dose of $10 \mathrm{mg} / \mathrm{kg}$ in 9 treated rats, whereas equal volume of PBS was iv injected in 6 control rats. For postmortem verifications of the in vivo imaging findings, 3 treated rats and 2 control rats were killed at 1 hour, 6 hours, and 2 days after the treatment immediately after the last MRI session as shown in the flow chart (Fig. 1).

\section{MR Imaging}

The rats were examined at a 1.5-Tesla whole body MR scanner (Sonata, Siemens, Erlangen, Germany) with a maximum gradient capability of $40 \mathrm{mT} / \mathrm{m}$ coupled with a 4-channel phased array wrist coil (MRI Devices, Waukesha, WI). The rats were placed

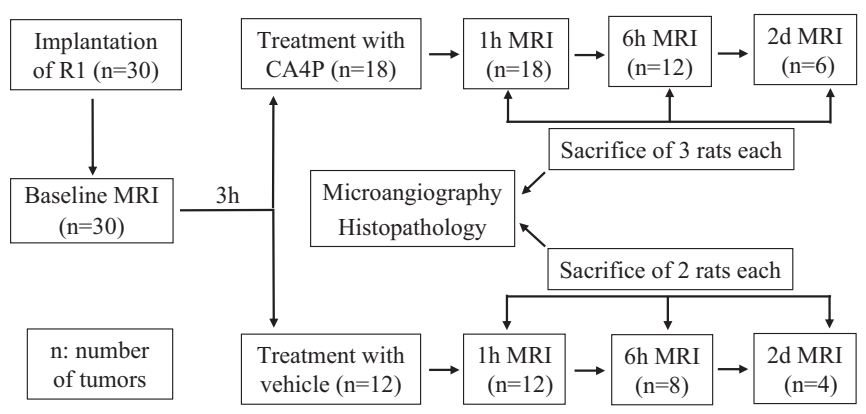

FIGURE 1. Flow chart of experimental protocol. supinely in a plastic holder with 2 lateral phantoms of tube containing $0.02 \%$ copper sulfate placed beside the trunk of rats as the external reference, providing high signal intensity on MR images for the normalization of signal intensity. The rat was worn a mask connected to a $10-\mathrm{m}$ polyethylene tube linked to a gas anesthesia system (Harvard Apparatus, Holliston, MA) outside the MRI suite. Initially the rats were anesthetized with inhalation of $5 \%$ isoflurane and then maintained through a mask with $2 \%$ isoflurane in the mixture of $20 \%$ oxygen and $80 \%$ room air. Before MR scanning, the tail vein of rat was cannulated for intravenous access.

After the pilot scan with axial, coronal, and sagittal images for localization, 12 axial images were obtained with a slice thickness of $3.5 \mathrm{~mm}$ and a gap of $0.7 \mathrm{~mm}$ for T1-(T1WI), T2-(T2WI), and diffusion weighted imaging sequences (DWI), T2*-weighted dynamic susceptibility imaging (DSI), and contrast enhanced T1WI (CE-T1WI).

For turbo spin-echo (SE) T1WI, the following parameters were used: repetition time/echo time of 457.0/8.6 milliseconds, turbo factor of 7 , field of view of $70 \times 140 \mathrm{~mm}$, imaging acquisition matrix of $128 \times 256$, and in-plane resolution of $0.55 \times 0.55 \mathrm{~mm}$. Four acquisitions led to a total data acquisition time of 1 minute 12 seconds.

For turbo-SE T2WI: repetition time/echo time of 2860/100 milliseconds, turbo factor of 19 , field of view of $70 \times 140 \mathrm{~mm}$, imaging acquisition matrix of $128 \times 256$, and in-plane resolution of $0.55 \times 0.55 \mathrm{~mm}$. Three acquisitions led to a total data acquisition time of 1 minute 4 seconds.

For DWI with 2-dimensional SE echo-planar imaging (EPI) sequence, a parallel imaging technique of generalized autocalibrating partially parallel acquisition (GRAPPA) was used with an acceleration factor of 2 to reduce the susceptibility artifacts and scanning time: repetition time/echo time of 1700/83 milliseconds, field of view of $82 \times 140 \mathrm{~mm}$, imaging acquisition matrix of $91 \times$ 192 , and in-plane resolution of $0.90 \times 0.73 \mathrm{~mm}$. Diffusion gradients were applied along 3 directions $(x, y$, and $z$ axes) with 10 different $b$ values $\left(0,50,100,150,200,250,300,500,750\right.$, and $\left.1000 \mathrm{~s} / \mathrm{mm}^{2}\right)$

For DSI, a T2*-weighted EPI sequence in combination with GRAPPA was used to increase spatial homogeneity on the images: repetition time/echo time of 2000/46 milliseconds, field of view of $70 \times 140 \mathrm{~mm}$, imaging acquisition matrix of $64 \times 128$, and in-plane resolution of $1.09 \times 1.09 \mathrm{~mm}$. The dynamic imaging series of 100 measurements resulted in a total examination time of 3 minutes 24 seconds. To ensure a sufficient number of precontrast baseline images, the iv bolus of gadodiamide (Omniscan; Amersham, Norway) at $0.3 \mathrm{mmol} / \mathrm{kg}$ was started after the 30 th measurement. The high dose of contrast agent was used to generate more prominent T2* effect on this DSI sequence. After DSI, each MRI session was ended up with a T1WI to obtain CE-T1WI taking the advantage of contrast agent for prior DSI.

The interval between the baseline-MRI and the treatment was kept 3 hours to ensure sufficient elimination of contrast agent from the blood and tissue.

\section{Digital Microangiography}

Immediately after the last MRI session, the rats were killed with an intravenous overdose of pentobarbital. Laparotomy was performed, and abdominal aorta was cannulated. Barium sulfate suspension (Micropaque; Guerbet, Roissy, Cedex, France) was injected via hepatic artery. Then the entire tumor-bearing liver was excised. With a digital mammographic unit (Embrace; Agfa-Gevaert, Mortsel, Belgium) at $25 \mathrm{kV}$ and $10 \mathrm{~mA}$, postmortem hepatic arteriography was made to document any changes in tumor vascularity. ${ }^{25}$ The injected barium particles also served as a greenyellowish intravascular markers in histopathological preparations. 
For quantification of vascular density, the fixed liver specimen was sliced in $3 \mathrm{~mm}$ using a Plexiglas matrix and radiographed again with the same protocol in the digital mammographic unit to compare the vascular density between the viable tumor, treatment-induced necrosis, and normal liver.

\section{Histopathological Analysis}

The liver specimens were fixed in $10 \%$ formaldehyde solution and processed for histopathological examinations. After paraffin embedding, 5- $\mu \mathrm{m}$ sections of the liver specimen were made in the plane oriented to the axial MR images, followed by hematoxylin and eosin staining. All the sections were microscopically assessed by a pathologist with 20 years of experiences for the presence and extent of viable tumor cells, necrosis, and intratumoral vasculature. The photomicrographs were further colocalized with the corresponding MR images (Figs. 2-5). Autopsy was performed to examine any adverse effects in major visceral organs including the brain, heart, lung, kidney, spleen, and normal liver.

\section{Imaging Analyses}

The image analyses were performed off-line on the Siemens workstation using the built-in software by 3 authors with consensus to obtain the following measurables.

\section{Tumor Volume}

By using an operator-defined region of interest (ROI), the area of tumor was manually delineated on each tumor-containing slice. The total area of tumor for the slices was calculated after summation and then multiplied by the slice thickness plus gap to obtain the total tumor volume using the following formula:

Tumor Volume

$$
=\sum \text { Tumor area on each tumor containing slice }
$$

$$
\times(\text { Slice thickness }+ \text { Gap) }
$$

\section{Measurement and Normalization of Signal Intensity}

For the measurement of SI, the tumor was contoured with an operator-defined ROI on the central tumor-containing slice of the images with the average tumor SI being automatically generated. For the delineation of tumor on apparent diffusion coefficient (ADC) maps derived with DWI, ROIs were copied and pasted from tumorcontaining DWI.

For the normalization of SI using the phantoms, the baseline image for each MRI sequence was assigned as a standard reference, a ROI of 160 pixels was placed on each phantom and the SIs of 2
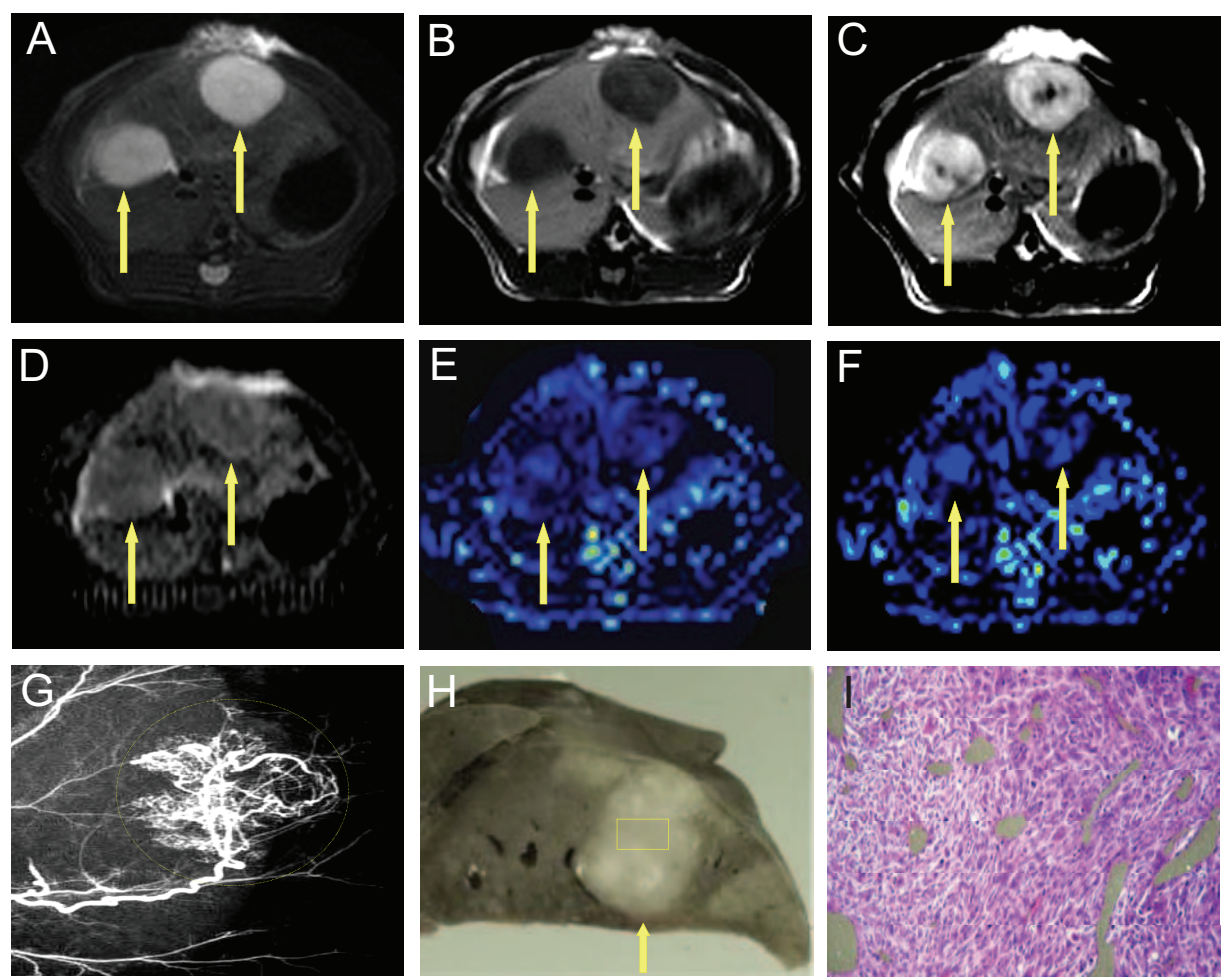

FIGURE 2. MRI and postmortem findings of rhabdomyosarcoma (R1) before intravenous injection of combretastatin A4-phosphate (CA4P) [A, T2Wl; B, T1Wl; C, contrast-enhanced T1WI (CE-TIWI); D, ADC; E, rBV; F, rBF; G, microangiography; H, macroscopy; and $\mathrm{I}$, histopathology]. The arrows denote the tumors. Relative to normal liver, the tumors appeared hyperintense on T2WI (A) and hypointense on T1WI (B) and was strongly enhanced with a clear border on CE-T1WI (C). On ADC map, the tumors appeared almost isointense to the normal liver, suggestive of viable tumor (D). The rBV (E) and rBF (F) of tumor were lower compared with normal liver because of solely arterial in the tumors and dual arterioportal blood supply in liver, respectively. On microangiography, the tumor (dotted circle) showed well developed hypertrophic and tortuous feeding arteries with irregular tumor staining (G). Macroscopic specimens showed the intrahepatic tumors as spheroid masses of a paler color growing in an expansion pattern against surrounding liver parenchyma $(\mathrm{H})$. Rectangular frame denotes the area where microscopy was focused. Microscopically, untreated liver masses were composed of viable R1 cells with large nuclei and numerous mitoses without cellular edema and vascular thrombosis. Tumoral vessels were filled with green-yellowish barium particles (I). 

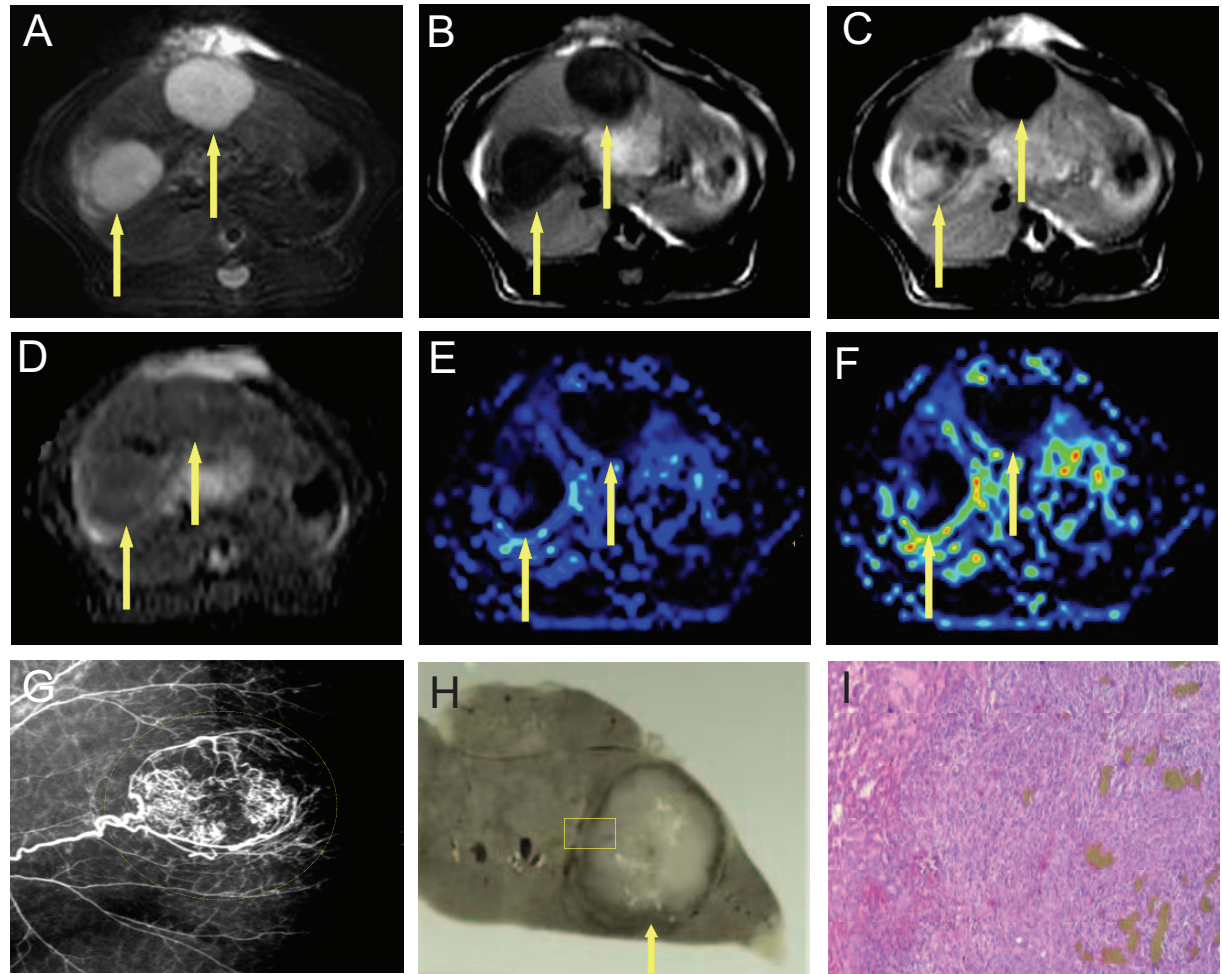

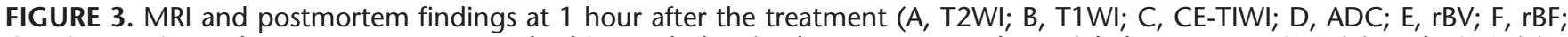
$\mathrm{G}$, microangiography; $\mathrm{H}$, macroscopy; and I, histopathology). There were no substantial changes on T2WI (A) and T1WI (B). On CE-T1WI, the tumors were hypointense relative to strongly enhanced normal liver, suggesting CA4P induced shutdown of intratumoral vasculature (C). Compared with baseline, the ADC value of tumor decreased slightly because of intracellular edema and impaired water mobility, suggestive of injured but viable tumoral tissues (D). The rBV (E) and rBF (F) decreased obviously. Besides the feeding artery, tumor staining (dotted circle) still existed, indicating in vivo functional vascular shutdown but postmortem patency for barium injection on microangiography (G), which explains the discrepant findings at 1 hour between in vivo MRI and postmortem microangiography (C, E, F vs. G). Tumor cells appeared viable but with pronounced edema. Without hemorrhage, tumoral blood vessels were congested with injected barium particles and suggests initial functional shutdown at 1 hour before permanent vascular thrombosis (I).

phantoms were averaged as a standard value. The normalized SI of each image was calculated with the following formula:

$\mathrm{SI}_{\text {normalized }}=\mathrm{SI}_{\text {measured }}$

$$
\times\left(\mathrm{SI}_{\text {phantom measured }} / \mathrm{SI}_{\text {phantom standard }}\right)
$$

The SI of tumor was graded as hypo-, iso-, or hyperintense relative to the SI of liver parenchyma.

\section{Estimation of Enhancement Ratio}

The following formula was used to compare the different degrees of enhancement after the injection of contrast agent at different time points.

Enhancement ratio $(\mathrm{ER})=\left(\mathrm{SI}_{\text {measured-post }}-\mathrm{SI}_{\text {measured-pre }}\right) /$

$$
\left(\mathrm{SI}_{\text {baseline-post }} / \mathrm{SI}_{\text {baseline-pre }}\right)
$$

where ER is enhancement ratio between the SI enhancement post-treatment relative to that at the baseline; baseline means pretreatment and measured means post-treatment; $\mathrm{SI}_{\text {measured-pre }}$ and $\mathrm{SI}_{\text {measured-post }}$ are the SI of tumor measured pre- and postcontrast at a certain time point after the treatment, respectively; and $\mathrm{SI}_{\text {baseline-pre }}$ and $\mathrm{SI}_{\text {baseline-post }}$ are the SI of tumor measured pre- and postcontrast at the baseline MRI before the treatment, respectively.

\section{Tumor Necrosis Ratio}

On CE-T1WI 2 days after CA4P administration, manual delineation of the area of necrosis and entire tumor was performed on the central tumor-containing slice with the areas of necrotic center and entire tumor automatically generated. The tumor necrosis ratio was defined as the area of necrotic center over that of the entire tumor.

\section{Relative Blood Volume and Relative Blood Flow Maps}

The DSI source images were processed with the software package (MR Neuro Perfusion Evaluation 1.0) built in the Siemens Sonata (NUMARIS/4 Syngo MR 2004A). Briefly, by placing a ROI located in the region of hepatic artery and aorta on DSI source images, the arterial input function was rendered by selecting 4 to 6 voxels containing artery elements that display the first-pass SI drop in the toolkit panel and then DSI-derived maps of relative blood volume $(\mathrm{rBV})$ and relative blood flow $(\mathrm{rBF})$ were generated. ${ }^{25}$ The calculation of $\mathrm{rBF}$ was performed using the standard deconvolution method as described by Ostergaard et al. ${ }^{27}$ For delineation of tumor and normal liver on the maps, ROIs were copied and pasted from the tumor- 
FIGURE 4. MRI and postmortem findings at 6 hours after the treatment (A, T2Wl; B, T1Wl; C, CETIWI; D, ADC; E, rBV; F, rBF; G, microangiography; $\mathrm{H}$, macroscopy; and I, histopathology). Compared with 1 hour, the tumors were still hypointense on CE-T1WI (C) and the $r B V(E)$ and $r B F(F)$ remained decreased, whereas the tumors turned slightly hyperintense on ADC map (D) as a sign for evolving to necrosis because of cell membrane breakdown and increased diffusion. On microangiography, nearly all the arteries disappeared in the center of tumor (dotted circle), and the tumor feeding arteries ended abruptly where they entered the tumor (G). Tumoral blood vessels were congested with stable thrombus. Tumor cells still appeared viable but with pronounced edema (I).
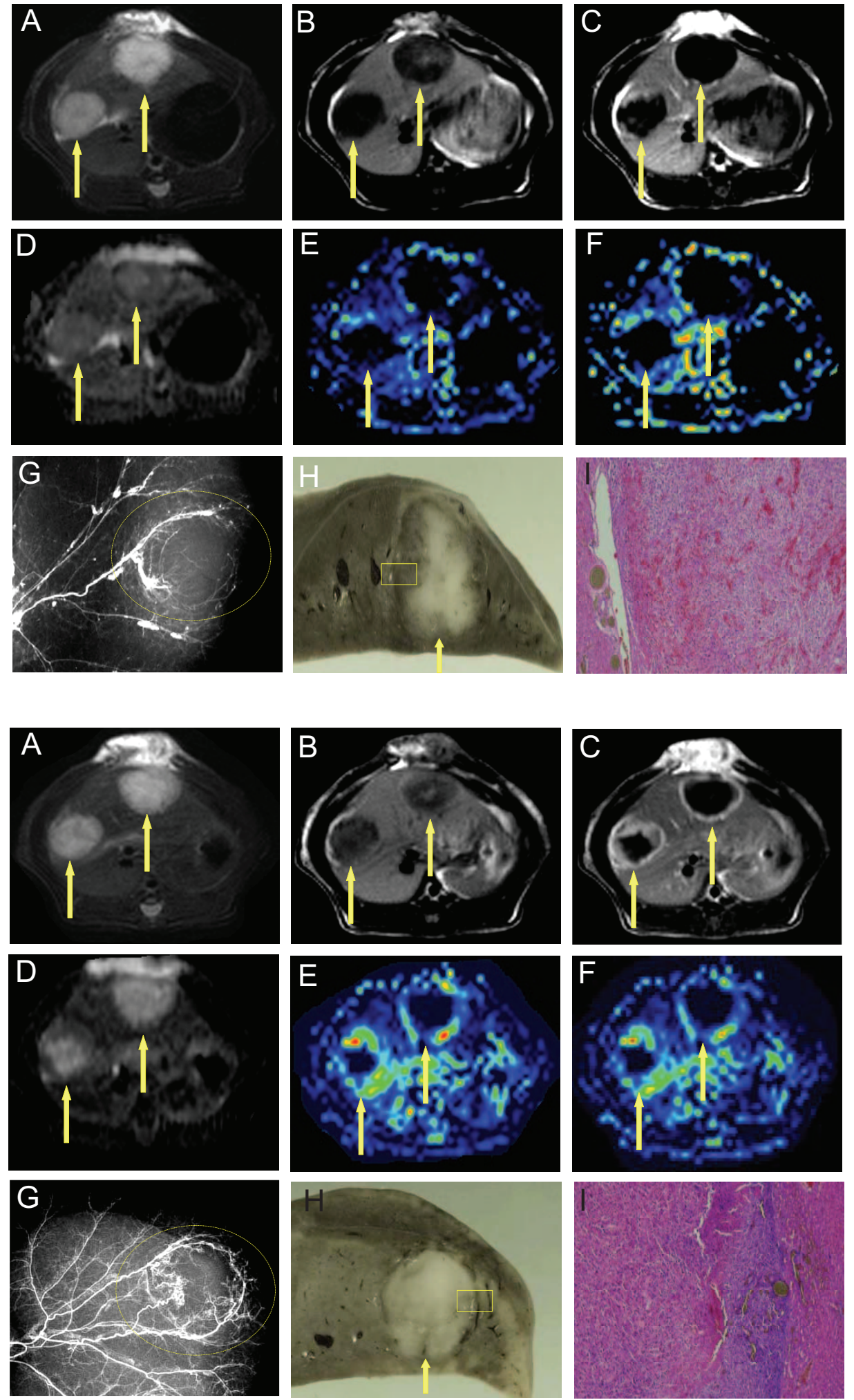

containing source image of DSI, and the values of rBV and rBF were automatically generated with arbitrary units (au). The values of the $\mathrm{rBV}$ and $\mathrm{rBF}$ for the tumor and normal liver post- and pretherapy were plotted to compare the drug effect on the different tissue components and at different time points. Local dynamic mean relative SI-time curves were obtained by placing the ROI on tumor and normal liver and converting the values in au into those relative to that of the baseline before contrast arrival. 


\section{Vascular Density on Microangiography}

After being transferred to a personal computer, the digitalized photographs of microangiography from the 3-mm slices were analyzed for their gray values with ImageJ (version 1.39, NIH). For the quantification of vascular density, the viable part in the periphery and necrosis in the center of tumor was delineated with operatordefined ROIs, and a round ROI about $1 \mathrm{~cm}^{2}$ was also drawn on the normal liver. The gray values were automatically generated and then compared between the viable tumor, treatment-induced necrosis, and normal liver.

\section{Statistical Analysis}

Statistical analysis was carried out with the Microsoft Excel 9.0 and SPSS for Windows software package (version 13.0; SPSS, Chicago, IL). Two-tailed Student $t$ test was used to compare the different variables in treated group and controls. Numerical data were reported as mean \pm standard deviation (SD). A significant difference was concluded if the $P$ value was less than 0.05 .

\section{RESULTS}

\section{General Conditions}

All animals survived the anesthesia, surgical procedures for tumor implantation, tumor development, and multiple MRI sessions. The bifocal liver R1 tumor model was successfully established in all rats. Because there was no overall significant difference between the volume of tumors located in left and right lobes $(P=0.71)$, the tumors in left and right liver lobes were merged into treated $(\mathrm{n}=18)$ or control $(\mathrm{n}=12)$ group for analyses. At 16 days after implantation and during this 2-day experiment, all rats $(n=15)$ appeared normal without pallor, piloerection, restlessness, weakness, tremors, diarrhea, ascites, and loss of hair or appetite, suggesting the absence of systemic disease.

\section{MRI Findings}

Based on MRI findings from the baseline to 2 days after the treatment (Figs. 2-5), CA4P significantly inhibited tumor growth at 2 days as shown with the tumor volume curves from the treated and control rats (Fig. 6)

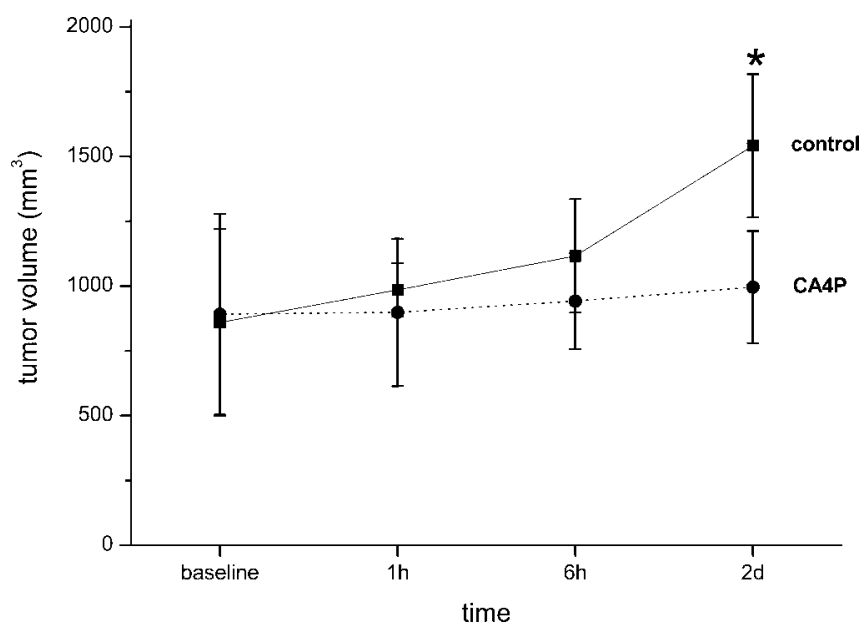

FIGURE 6. Line chart comparing tumor volumes at different time points between treated and control animals. Tumors in control group showed a pattern of accelerating growth, whereas tumors treated with CA4P grew significantly slower within 2 days. ${ }^{*} P<0.05$ for CA4P versus controls at day 2 .
On the baseline, all the tumors demonstrated a spherical shape with a clear border, easily demarcated from the normal liver with all imaging sequences. In the center of some tumors, minute dots or irregular foci of necrosis were noticed (Fig. 2).

On T2WI, the tumor showed homogeneous hyperintensity (Fig. 2A). On T1WI, the tumor appeared hypointense (Fig. 2B). On CE-T1WI, the tumor showed a homogeneous enhancement at baseline but various degrees of enhancement because of the drug effects and tumor response from 1 hour to 2 days in treated group (Figs. 2C, 3C, 4C, 5C). By contrast, all the tumors in control group shared the same MRI characteristics from the baseline to 2 days after the treatment, except the increasing tumor size.

\section{Tumor Growth with and without CA4P Treatment}

The average tumor volume turned out to be $892 \pm 387 \mathrm{~mm}^{3}$ in treated group and $860 \pm 278 \mathrm{~mm}^{3}$ in control group at baseline $(P$ $=0.67)$. The tumors in control group grew rapidly from 1 hours to 2 days: $985 \pm 103 \mathrm{~mm}^{3}$ at 1 hour, $1116 \pm 219 \mathrm{~mm}^{3}$ at 6 hours, and 1542 $\pm 277 \mathrm{~mm}^{3}$ at day $2(P<0.05,2$ days vs. baseline, 1 hour and 6 hours $)$. The tumors in CA4P-treated group showed a slower growth: $899 \pm$ $284 \mathrm{~mm}^{3}$ at 1 hour, $942 \pm 185 \mathrm{~mm}^{3}$ at 6 hours, and $996 \pm 216 \mathrm{~mm}^{3}$ at day 2 without significant difference from the baseline to day $2(P$ $>0.05$ ). However, tumor volume was significantly smaller in the treated group compared with the control group at 2 days $(P<0.05)$ as shown in Figures 2 to 6.

\section{Contrast-Enhanced T1WI}

In contrast to a strong signal enhancement throughout the entire tumor at the baseline and in control group, except some minute unenhanced necroses (Fig. 2C), most of the tumors showed absence of enhancement at 1 hour after CA4P treatment because of the shutdown of intratumoral blood flow (Fig. 3C), which evolved into a more complete effect at 6 hours (Fig. 4C). At day 2, an enhanced ring was shown in tumor periphery, whereas the central region remained unenhanced (Fig. 5C).

\section{Enhancement Ratio}

Compared with the ER of $1.00 \pm 0.00$ on the baseline, the ER dropped significantly to $0.37 \pm 0.25(P<0.01)$ at 1 hour and 0.26 \pm 0.22 at 6 hours $(P>0.05$ vs. 1 hour $)$ but significantly recovered to $0.55 \pm 0.16(P<0.01$ vs. 6 hours $)$ at 2 days after treatment in treated group. Such alterations were absent in control group.

\section{Necrosis Ratio}

Contrary to the absence of any change in the control group, the tumor necrosis ratio in the treated group was significantly increased from $0.02 \pm 0.06$ at baseline to $0.71 \pm 0.16(P<0.01)$ at day 2 in the treated group. Because tumor necrosis was not histologically evident, this ratio was not calculated at 1 hour and 6 hours.

\section{ADC Maps}

On DWI-derived ADC maps, the tumors appeared almost isointense on the baseline (Fig. 2D), slightly hypointense at 1 hour (Fig. 3D) and slightly hyperintense at 6 hours (Fig. 4D). The mean ADC of tumors on baseline was $1.10 \times 10^{-3} \pm 0.14 \times 10^{-3} \mathrm{~mm} / \mathrm{s}^{2}$. After CA4P administration, it decreased to $0.96 \times 10^{-3} \pm 0.16 \times$ $10^{-3} \mathrm{~mm} / \mathrm{s}^{2}$ at 1 hours $(P<0.05$ vs. the baseline) and to $1.00 \times$ $10^{-3} \pm 0.14 \times 10^{-3} \mathrm{~mm} / \mathrm{s}^{2}$ at 6 hours $(P>0.05$ between 1 hours and 6 hours). At day 2, the central region showed hyperintense, whereas the periphery remained almost isointense (Fig. 5D). The mean $\mathrm{ADC}$ increased to $1.38 \times 10^{-3} \pm 0.22 \times 10^{-3} \mathrm{~mm} / \mathrm{s}^{2}$ $(P<0.01$, vs. baseline, 1 hours and 6 hours $)$. No similar alterations were detected in tumors of the control group. 
FIGURE 7. Bar chart comparing the rBV (A) and $\mathrm{rBF}(\mathrm{B})$ of tumor and liver in CA4P-treated animals shows a decrease from 1 hour to 6 hours, but a rebound at day 2 only in tumors, rather than in the liver after drug administration. ${ }^{*} P<$ 0.05 versus the previous time point.
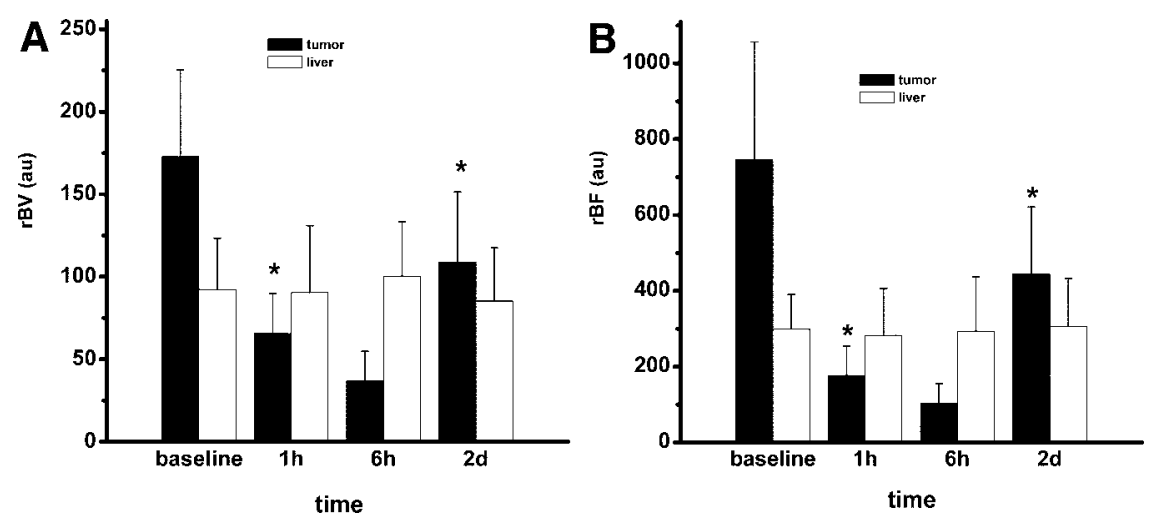
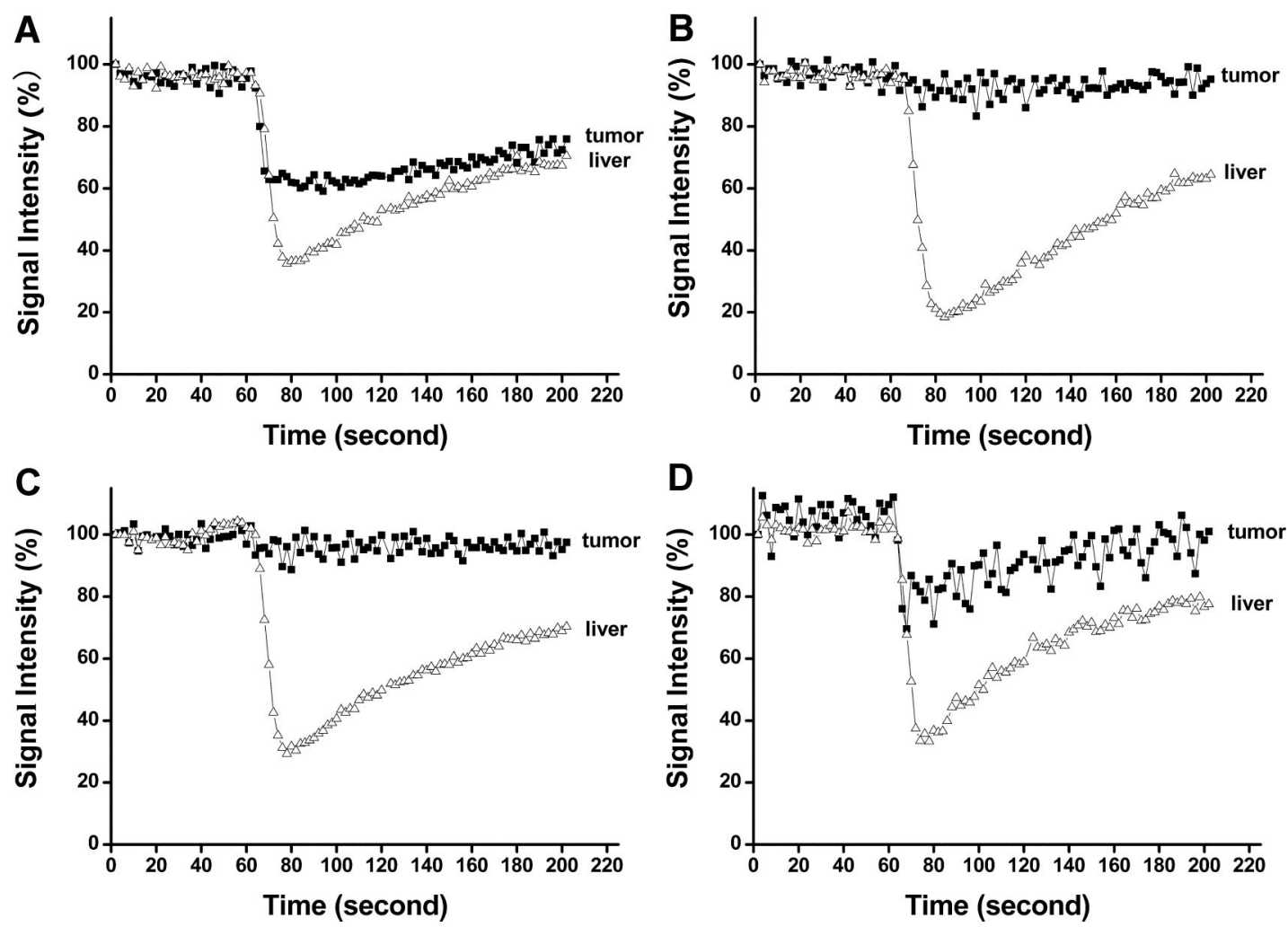

FIGURE 8. The dynamic mean relative SI-time curves derived from T2*-weighted dynamic susceptibility imaging (DSI) show a signal drop during the first pass in both the tumor and normal liver of the control animals and at the baseline of the CA4Ptreated animals (A). At 1 hour (B) and 6 hours (C) after CA4P administration in the CA4P-treated group, this first-pass effect disappeared only with the tumors but not with the normal liver, suggesting a tumor-selective effect of this drug. However, at day 2 (D), the tumoral signal drop partially recovered, suggesting the peripheral tumor recurrence.

\section{Dynamic Susceptibility Imaging}

Figure 7 shows the rBV and rBF of the tumor and normal liver. These tumor blood perfusion parameters showed a significant decrease from baseline (Figs. 2E, F) to 1 hours (Figs. 3E, F), a continuous decrease at 6 hours (Figs. 4E, F), and a significant recovery at day 2 after CA4P treatment (Figs. 5E, F and 7). No significant difference was shown between 1 hour and 6 hours. Such evolutions never occurred in control group.

The mean relative SI-time curves for the tumor and normal liver derived from the DSI source images are shown in Figure 8. On the baseline, the curves for the tumor and normal liver shared the same pattern of first-pass T2* effect though to different extents (Fig.
8A). However, such an effect disappeared only with the tumor but not with normal liver at 1 hour and 6 hours after the CA4P treatment (Figs. 8B, C). At day 2, the first pass effect was partially recovered for the tumor (Fig. 8D).

\section{Microangiographic Findings}

The representative tumors obtained at different time points from the control group showed well-developed hypertrophic and tortuous feeding arteries with irregular tumor staining (Fig. 2G). In treated group, at 1 hour after CA4P administration, the tumorfeeding arteries and tumor staining still existed (Fig. 3G), which appeared contradictory to the MRI findings but suggests that the 
vascular shutdown was just functional at this acute phase, for the tumoral vessels could still be filled with injected barium. At 6 hours, nearly all the intratumoral vessels disappeared particularly in the center and the feeding arteries ended abruptly at the site where they would enter the tumor (Fig. 4G). At day 2, no vessel was opacified in the center of tumor, whereas abundant neovasculature emerged at the periphery of the tumor (Fig. 5G). These microangiographic features fully conform to MRI findings (Figs. 2C, E, F; 3C, E, F; 4C, E, F; 5C, E, F).

Quantitatively, the gray value as measured with 3-mm-thick slice of microangiography was $139.1 \pm 32.2$ for viable tumor, $79.5 \pm$ 8.6 for normal liver, and $37.0 \pm 8.8$ for necrosis. The vascular density of viable tumor was significantly higher than the necrosis or normal liver $(P<0.05)$.

\section{Histopathological Findings}

At autopsy, no intrahepatic and remote metastases and no anomaly of the liver and other organs were found in both groups of rats. Macroscopically, the intrahepatic R1 masses grew in an expansion pattern against liver parenchyma with a paler color than surrounding normal liver (Figs. $2 \mathrm{H}, 3 \mathrm{H}, 4 \mathrm{H}, 5 \mathrm{H}$ ). Microscopically, liver masses in the control group were composed of viable rhabdomyosarcoma cells with large nuclei and numerous mitoses. Small necrotic foci were occasionally shown in the center of tumor. Abundant tumoral vasculature was filled with green-yellowish barium particles and no cellular edema and vascular thrombosis were present (Fig. 2I)

At 1 hour and 6 hours after the CA4P administration, pronounced cellular edema and congested blood vessels without hemorrhage were observed in the center of tumors, though tumor cells appeared viable (Figs. 3I, 4I). A major difference between these 2 time points is that the intratumoral vessels were filled with barium particles at 1 hour (Fig. 3I) but with stable thrombus at 6 hours (Fig. 4I), which explains the discrepant findings at 1 hour between in vivo MRI and postmortem microangiography (Figs. 3B-F vs. 3G) and suggests initial functional shutdown at 1 hour before permanent vascular occlusion at 6 hours by the stable clots. At day 2 , massive central necrosis with peripheral calcification occurred in the tumor. Meanwhile, the new blood vessels proliferated and supplied blood to the outer layers of viable tumor cells with vigorous mitotic phases (Fig. 5I). No visible abnormalities were observed in the specimens of the major organs from both groups.

\section{DISCUSSION}

The present study has unambiguously demonstrated the potent selective therapeutic effect of CA4P on rodent malignant liver tumors and the feasibility of using a clinically available $1.5-\mathrm{T}$ MRI scanner to monitor and evaluate intratumoral therapeutic events. To our knowledge, it is the first time that the effect of systemic chemotherapy on visceral tumors could be vividly displayed within hours using noninvasive MRI. The experimental data thus derived, which otherwise would be impossible to obtain from human patients, can bridge in vivo and ex vivo findings, correlate morphologic and functional features, and eventually broaden our knowledge about emerging anticancer drugs.

\section{Rodent Model of Liver Rhabdomyosarcoma}

Tumors, even originated from the same cell line, might differ in their biologic behaviors, growing rates, and responses to the same therapeutic regimen, if residing in different target organs. The reason lies in the fact that nutrients and oxygen delivery to the tumors vary greatly among organs with different levels of vascularization. ${ }^{28}$ Consequently, subcutaneous implantation of R1 tumor might not fully present its true biology compared with a visceral R1 growth. Compared with subcutaneous implantation, intrahepatic growth of R1 is more clinically relevant in terms of deep-located tumors in visceral organs as more commonly seen in patients. ${ }^{8,17,23,24}$ Liver is the largest parenchymal organ that harbors various primary and metastatic malignancies. By means of implantation of tumor into the liver, this liver tumor model could better mimic the features of human liver metastasis ${ }^{25,29}$ for conducting more clinically translational research as exemplified by the present study.

\section{Response of Liver Tumor to CA4P}

As a VTA, CA4P has shown its efficacy for selectively reducing tumoral blood flow in phase I clinical trials and is being evaluated in phase II clinical trials in combination with conventional cytotoxic therapies for the potential treatment of solid cancers. ${ }^{30} \mathrm{In}$ the present study, such therapeutic outcomes could be directly visualized by in vivo MRI and proven by postmortem microangiography and histopathology. However, as clearly demonstrated here, CA4P was only able to inhibit tumor growth but neither to eradicate the entire tumor nor to reduce the tumor volume because of peripheral tumor residues and subsequent tumor recurrence. This may explain the suboptimal clinical outcomes to date with VTAs despite over 2 decades of drug development. Therefore, various new regimes have been proposed to improve clinical outcomes, including repeated dosages of $\mathrm{CA} 4 \mathrm{P}^{31}$ and combined use of hyperthermia, ${ }^{32}$ radiation, ${ }^{33}$ or other conventional chemotherapy. ${ }^{34}$

A solid tumor is not just a collection of cancer cells but forms a complex microenvironment with 3 basic components, ie, cancer cells, blood vessels, and interstitia. Tumors depend on neoangiogenesis to survive beyond a volume of a few cubic millimeters. Smaller tumors derive their blood supply from adjacent tissues with normal vascular endothelia, which will not be attacked by CA4P. In a growing larger tumor, however, 2 different vessels exist: $(a)$ preexisting normal host vessels usually dislocated to the periphery because of tumoral expansion and $(b)$ newly formed aberrant vessels with abnormal vascular endothelia typically distributed inside the tumor mass. Besides, peripheral tumor cells can acquire nutrient and oxygen diffusing from adjacent normal tissue. These inherent morphologic and biologic properties of solid tumors determine the observed common features in CA4P treatment, ie, (1) large tumors are attacked more effectively than small ones ${ }^{8}$; (2) central part appears more vulnerable than peripheral part of the tumor to the drug effect ${ }^{7}$; and (3) consequently, tumor recurrence occurs almost always from the periphery, as demonstrated with the significantly higher vascular density of viable tumor at 2 days after the treatment However, early clinical differentiation between residual tumor and peritumoral tissue reaction after cancer therapy can be problematic.

Practically, we applied iv administration of CA4P at the clinically relevant dose of $10 \mathrm{mg} / \mathrm{kg}$, which gave a plasma exposure close to that achieved in humans using the maximal tolerated dose of $65 \mathrm{mg} / \mathrm{m}^{2}$ in the clinical trials. ${ }^{7}$ This treatment regimen showed selective anticancer effects without detectable toxic effects on the normal liver and other organs. However, 1 single dosage of CA4P could not obtain a sustained effect, and tumor recurrence occurred within a very short period. The insight thus obtained may provide hints for further improvements on this promising therapy.

\section{Crucial Roles of Advanced MRI with Functional Parameters}

In this study, we were able to perform noninvasive and longitudinal investigations to visualize and quantify the therapeutic events in a rodent liver tumor model, thanks to the recent advances in development of MRI sequences that enable 2-D and 3-D highresolution images and integrate both morphologic and functional information. Conventional T1WI and T2WI are insufficient to unambiguously demarcate lesional and normal tissues and to distin- 
guish viable and necrotic tumor components, whereas CE-T1WI may underestimate necrosis because of diffusion of the contrast agent. $^{21}$

DCE-MRI, on the other hand, has been applied for evaluation of tumor perfusion with 2 basic methods, namely using T1- and T2*-weighting. T1-weighted DCE-MRI can be assessed using either semiquantitative, such as initial slope and area under the curve, or quantitative measures, such as $K^{\text {trans }} .35,36$ However, another approach that has shown promise is adopting the T2*-weighted DSI quantification methods of the brain to a rat model of intrahepatic R1 implantation. ${ }^{25}$ During the first pass of contrast agent, the susceptibility changes in the capillary network can be detected as a transient signal drop on $\mathrm{T} 2 *$-weighted images. ${ }^{37}$ In the present study by using the built-in software, perfusion-related parameters such as $\mathrm{rBV}$ and $\mathrm{rBF}$ before and after treatment could be quantified to reflect the drug-induced dynamic alterations of tumoral blood supply, as verified by microangiography and histopathology. Based on the present study, blood flow (rBF) seems more relevant than blood volume (rBV) to post-therapeutic assessment of such anticancer therapies. SI-time curves also clearly revealed discrepant responses between tumoral and normal vasculature toward the effect of CA4P.

The DSI-derived rBV and rBF maps successfully demonstrated the significant post-treatment decrease in blood flow and blood volume at 1 hour and more evident at 6 hours, and the partial recovery at 2 days after the administration of CA4P. These evolutional changes of blood supply were concordant with the findings in the study of a mouse subcutaneous allograft tumor model treated with a retroorbital injection of CA4P analogue, AVE8062, performed with dynamic contrast-enhanced ultrasonography. ${ }^{24}$

DWI and related ADC maps exploit the random microscopic motion of water protons, a parameter reflecting tissue viability because of the physical and structural diversity between viable and dead tissues. ${ }^{38}$ Viable tumors have intact cell membrane that restricts water motility, whereas necrotic tumors are characterized by the increased permeability of tissue and diffusion of water molecules resulting from breakdown of cell membrane. Having been widely used for characterization of tumors and evaluation of tumor response to therapies, these techniques prove particularly critical for therapeutic assessment of VTAs such as CA4P because these drugs only inhibit tumor growth but do not reduce tumor volume. Although DWI has been applied in experiments with CA4P in subcutaneous tumor models, ${ }^{17}$ to our knowledge, the present study is the first one to apply this technique for evaluation of CA4P in a rodent liver tumor model. Geschwind et $\mathrm{al}^{20}$ reported the use of DWI in a rabbit liver tumor model, which was performed shortly after killing the animal to prevent motion artifacts. By optimizing acquisition parameters, the motion artifacts were minimized in the present setting. ${ }^{25}$

ADC values reflect tumoral cell density, which is complementary to the information about tumoral vascular supplies derived from DCE-MRI. ${ }^{21}$ Low and high ADC may refer to higher and lower cellular density, increased and reduced diffusion barriers, and lessened and raised water proton motility in the viable and nonviable tissues, respectively. ${ }^{39}$ These principles explain well the observed evolving intratumoral events caused by CA4P in the present study. Compared with baseline, ADC initially decreased at 1 hour after therapy. At this point, the tumor cells swelled because of vascular shutdown and hypoxia, resulting in decreased extracellular space and impeded water proton motions, whereas the tumor cells still remained viable as proven by histopathology. Starting from 6 hours, ADC gradually increased and became more prominent at day 2 because of enlarged extracellular space resulting from the breakdown of cell membrane and subsequent central necrosis of the tumor. The periphery with lower ADC at day 2 corresponded to the recurrent viable tumor tissues. Such information for differentiation of viable from necrotic tumor would be impossible to obtain if conventional MRI sequences such as T2WI, T1WI, and CE-T1WI were used only.

\section{STUDY LIMITATIONS}

There exist a number of limitations in this study. First, we applied a rodent R1 liver tumor model as the only subject for the therapy, which may limit the scope of the study conclusions. However, similar results on CA4P have been reported from the subjects with other tumor types in both clinical and experimental settings though mostly by less robust MRI methods. ${ }^{12,40}$ Second, we monitored the therapeutic effects of CA4P only for 2 days after drug administration without a long-term follow-up to determine animal survival as an end point. However, this acute phase has enabled us to document the most dramatic events of tumor responses to CA4P and the early tumor recurrence as a clear sign for poor prognosis. Third, although by using DCE-MRI with clinically available software, we were able to quantitatively measure the blood perfusion-related parameters, which are particularly crucial for studying VTAs, the accuracy of the method was not validated by other more delicate in vivo techniques such as Doppler ultrasound and direct flowmetric measurement. These shortcomings will be addressed in our ongoing research.

In conclusion, CA4P proves to be an effective VTA to cause rapid vascular shutdown in this rodent liver tumor model, although tumor recurrence at the periphery seems unavoidable if the drug is used alone. The therapeutic effects of CA4P can be noninvasively monitored with multiparametric MRI. DWI and DCE-MRI sequences provide potent measures for assessment of anticancer treatment.

\section{Practical Application}

Noninvasive devascularization can be achieved by intravenous administration of vascular targeting agent CA4P at a clinically equivalent dose for the treatment of rodent liver malignancies, though with evident tumor peripheral residue and recurrence. The in vivo intratumoral events after therapy can be monitored by using a clinical 1.5-T MRI scanner for both morphologic and functional evaluations.

\section{REFERENCES}

1. Thorpe PE. Vascular targeting agents as cancer therapeutics. Clin Cancer Res 2004; 10:415-427.

2. Siemann DW, Chaplin DJ, Horsman MR. Vascular-targeting therapies for treatment of malignant disease. Cancer. 2004;100:2491-2499.

3. Pettit GR, Singh SB, Hamel E, et al. Isolation and structure of the strong cell growth and tubulin inhibitor combretastatin A-4. Experientia. 1989;45:209211 .

4. Tozer GM, Kanthou C, Parkins CS, et al. The biology of the combretastatins as tumour vascular targeting agents. Int J Exp Pathol. 2002;83:21-38.

5. Woods JA, Hadfield JA, Pettit GR, et al. The interaction with tubulin of a series of stilbenes based on combretastatin A-4. Br J Cancer. 1995;71:705711

6. West CM, Price P. Combretastatin A4 phosphate. Anticancer Drugs. 2004; 15:179-187.

7. Prise VE, Honess DJ, Stratford MR, et al. The vascular response of tumor and normal tissues in the rat to the vascular targeting agent, combretastatin A-4-phosphate, at clinically relevant doses. Int J Oncol. 2002;21:717-726.

8. Landuyt W, Verdoes O, Darius DO, et al. Vascular targeting of solid tumours a major 'inverse' volume-response relationship following combretastatin A-4 phosphate treatment of rat rhabdomyosarcomas. Eur J Cancer. 2000;36: 1833-1843.

9. Galbraith SM, Maxwell RJ, Lodge MA, et al. Combretastatin A4 phosphate has tumor antivascular activity in rat and man as demonstrated by dynamic magnetic resonance imaging. J Clin Oncol. 2003;21:2831-2842.

10. Tozer GM, Prise VE, Wilson J, et al. Combretastatin A-4 phosphate as tumor vascular-targeting agent: early effects in tumors and normal tissues. Cancer Res. 1999;59:1626-1634. 
11. Rustin GJ, Galbraith SM, Anderson H, et al. Phase I clinical trial of weekly combretastatin A4 phosphate: clinical and pharmacokinetic results. $J$ Clin Oncol. 2003;21:2815-2822.

12. Dowlati A, Robertson K, Cooney M, et al. A phase I pharmacokinetic and translational study of the novel vascular targeting agent combretastatin A-4 phosphate on a single-dose intravenous schedule in patients with advanced cancer. Cancer Res. 2002;62:3408-3416.

13. Stevenson JP, Rosen M, Sun W, et al. Phase I trial of the antivascular agent combretastatin A4 phosphate on a 5-day schedule to patients with cancer: magnetic resonance imaging evidence for altered tumor blood flow. J Clin Oncol. 2003;21:4428-4438.

14. Kirwan IG, Loadman PM, Swaine DJ, et al. Comparative preclinical pharmacokinetic and metabolic studies of the combretastatin prodrugs combretastatin A4 phosphate and A1 phosphate. Clin Cancer Res. 2004;10:14461453.

15. Anderson HL, Yap JT, Miller MP, et al. Assessment of pharmacodynamic vascular response in a phase I trial of combretastatin A4 phosphate. J Clin Oncol. 2003;21:2823-2830.

16. Nambu H, Nambu R, Melia M, et al. Combretastatin A-4 phosphate suppresses development and induces regression of choroidal neovascularization. Invest Ophthalmol Vis Sci. 2003;44:3650-3655.

17. Thoeny HC, De Keyzer F, Chen F, et al. Diffusion-weighted MR imaging in monitoring the effect of a vascular targeting agent on rhabdomyosarcoma in rats. Radiology. 2005;234:756-764.

18. Valonen PK, Lehtimaki KK, Vaisanen TH, et al. Water diffusion in a rat glioma during ganciclovir-thymidine kinase gene therapy-induced programmed cell death in vivo: correlation with cell density. J Magn Reson Imaging. 2004;19:389-396.

19. Roth Y, Tichler T, Kostenich G, et al. High-b-value diffusion-weighted MR imaging for pretreatment prediction and early monitoring of tumor response to therapy in mice. Radiology. 2004;232:685-692.

20. Geschwind JF, Artemov D, Abraham S, et al. Chemoembolization of liver tumor in a rabbit model: assessment of tumor cell death with diffusionweighted MR imaging and histologic analysis. J Vasc Interv Radiol. 2000; 11:1245-1255.

21. Lang P, Wendland MF, Saeed M, et al. Osteogenic sarcoma: noninvasive in vivo assessment of tumor necrosis with diffusion-weighted MR imaging. Radiology. 1998;206:227-235.

22. Kamel IR, Bluemke DA, Ramsey D, et al. Role of diffusion-weighted imaging in estimating tumor necrosis after chemoembolization of hepatocellular carcinoma. AJR Am J Roentgenol. 2003;181:708-710.

23. Thoeny HC, De Keyzer F, Vandecaveye V, et al. Effect of vascular targeting agent in rat tumor model: dynamic contrast-enhanced versus diffusionweighted MR imaging. Radiology. 2005;237:492-499.

24. Lavisse S, Lejeune P, Rouffiac V, et al. Early quantitative evaluation of a tumor vasculature disruptive agent AVE8062 using dynamic contrast-enhanced ultrasonography. Invest Radiol. 2008;43:100-111.

25. Chen F, Sun X, De Keyzer F, et al. Liver tumor model with implanted rhabdomyosarcoma in rats: MR imaging, microangiography, and histopathologic analysis. Radiology. 2006;239:554-562.

26. Hermens AF, Barendsen GW. Cellular proliferation patterns in an experimental rhabdomyosarcoma in the rat. Eur J Cancer. 1967;3:361-369.

27. Ostergaard L, Weisskoff RM, Chesler DA, et al. High resolution measurement of cerebral blood flow using intravascular tracer bolus passages. Part I: mathematical approach and statistical analysis. Magn Reson Med. 1996;36: 715-725.

28. Fidler IJ. Critical determinants of metastasis. Semin Cancer Biol. 2002;12 89-96.

29. Wang H, Van de Putte M, Chen F, et al. Murine liver implantation of radiation-induced fibrosarcoma: characterization with MR imaging, microangiography and histopathology. Eur Radiol. 2008;18:422-430.

30. Chaplin DJ, Horsman MR, Siemann DW. Current development status of small-molecule vascular disrupting agents. Curr Opin Investig Drugs. 2006; 7:522-528

31. Thoeny HC, De Keyzer F, Chen F, et al. Diffusion-weighted magnetic resonance imaging allows noninvasive in vivo monitoring of the effects of combretastatin a-4 phosphate after repeated administration. Neoplasia. 2005 7:779-787.

32. Murata R, Overgaard J, Horsman MR. Combretastatin A-4 disodium phosphate: a vascular targeting agent that improves that improves the anti-tumo effects of hyperthermia, radiation, and mild thermoradiotherapy. Int J Radiat Oncol Biol Phys. 2001;51:1018-1024.

33. Landuyt W, Ahmed B, Nuyts S, et al. In vivo antitumor effect of vascular targeting combined with either ionizing radiation or anti-angiogenesis treatment. Int J Radiat Oncol Biol Phys. 2001;49:443-450.

34. Siemann DW, Mercer E, Lepler S, et al. Vascular targeting agents enhance chemotherapeutic agent activities in solid tumor therapy. Int $J$ Cancer. 2002;99:1-6.

35. Padhani AR. Dynamic contrast-enhanced MRI in clinical oncology: current status and future directions. J Magn Reson Imaging. 2002;16:407-422.

36. Cyran CC, Sennino B, Chaopathomkul B, et al. Magnetic resonance imaging assays for dimethyl sulfoxide effect on cancer vasculature. Invest Radiol. 2008;43:298-305

37. Heiland S, Kreibich W, Reith W, et al. Comparison of echo-planar sequences for perfusion-weighted MRI: which is best? Neuroradiology. 1998;40:216221

38. Bammer R. Basic principles of diffusion-weighted imaging. Eur J Radiol. 2003;45:169-184.

39. Lyng H, Haraldseth O, Rofstad EK. Measurement of cell density and necrotic fraction in human melanoma xenografts by diffusion weighted magnetic resonance imaging. Magn Reson Med. 2000;43:828-836.

40. Beauregard DA, Pedley RB, Hill SA, et al. Differential sensitivity of two adenocarcinoma xenografts to the anti-vascular drugs combretastatin A4 phosphate and 5,6-dimethylxanthenone-4-acetic acid, assessed using MRI and MRS. NMR Biomed. 2002;15:99-105. 